\title{
Opto-thermo-mechanical numerical simulations of 3 different concepts of infrared achromatic phase shifters
}

Bruno Chazelas $^{a}$, Claude Valette ${ }^{a}$, Thierry Lépine $^{b}$, Marc Barillot $^{d}$, Frank Brachet ${ }^{a}$, Sebastien Dervaux $^{c}$, François Fressin ${ }^{c}$, Jean Gay ${ }^{c}$, Gregory Gadret ${ }^{f}$, Alain Labèque ${ }^{a}$, Alain Leger ${ }^{a}$, Ralph Launhardt $^{h}$, Jacques Mangin ${ }^{f}$, Dimitri Mawet ${ }^{g}$, Marc Ollivier $^{a}$, Yves Rabbia ${ }^{c}$, Elke Schmidt ${ }^{e}$

${ }^{a}$ Institut d'Astrophysique Spatiale, bat 121, CNRS, Univ. Paris-Sud, F-91405 Orsay, France

${ }^{b}$ Institut d'Optique, Orsay/St-Etienne, France ; ${ }^{c}$ Observatoire de la Côte d'Azur, France ${ }^{d}$ Alcatel Space, France; ${ }^{e}$ Kayser Threde, Germany ; ${ }^{\circ}$ LPUB, Université de Bourgogne, France ${ }^{g}$ Université de Liège, Belgium ; ${ }^{h}$ MPIA

\begin{abstract}
The Darwin/TPF mission aims at detecting directly extra solar planets. It is based on the nulling interferometry, concept proposed by Bracewell in 1978, and developed since 1995 in several European and American laboratories. One of the key optical devices for this technique is the achromatic phase shifter (APS). This optical component is designed to produce a $\pi$ phase shift over the whole Darwin spectral range (i.e. $6-18 \mu \mathrm{m}$ ), and will be experimentally tested on the NULLTIMATE consortium nulling test bench (Labèque et al). ${ }^{1}$ Three different concepts of APS are being simulated: dispersive plates focus crossing and field reversal. In this paper, we show how thermal, mechanical and optical models are merged into a single robust model, allowing a global numerical simulation of the optical component performances. We show how these simulations help us to optimizing the design and present results of the numerical model.
\end{abstract}

Keywords: Darwin, numerical simulation, achromatic phase shifter, Nulling, Interferometry

\section{INTRODUCTION}

The current concept of the Darwin instrument is based on nulling interferometry. This technique requires an achromatic phase shifter (APS) over the Darwin spectral range $(6-18 \mu \mathrm{m})$. This device produce a $\pi$ phase shift over the whole spectral band. The European Space Agency has charged the NULLTIMATE consortium* to test experimentally and simulate three different concepts of APS (See Figure 1). The consortium has already selected the concepts that will be breadboarded :

- An APS based on the focus crossing principle (APSFC) : A well known property of a converging beam (Gouy effect) is that after the focal point the phase is $\pi$ shifted, whatever the wavelength. This device takes advantage of this property.

- An APS based on the field reversal technique (APSFR) : This device, is a clever geometrical arrangement return the electrical field of one arm of an interferometer compared to the other.

- An APS based on the dispersive plate concept (APSDP) : This device is based on the same principle than achromats, the selection of material combinations whose dispersion mutually compensates through the whole spectral band.

This paper will outline the simulation methods of the three APS and the first results of the modeling. The experimental set up is described in these proceedings in Labèque et al ${ }^{1}$ It will be a cryogenic tank working at $100^{\circ} \mathrm{K}$. The simulation described in this paper intend to help the interpretation of the experimental results that will be obtained on the NULLTIMATE Test Bench. It should also help the dimensioning of the APS and the test facility, particularly regarding the thermal and vibration point.

Further author information: (Send correspondence to Bruno Chazelas)

Bruno Chazelas.: E-mail: bruno.chazelas@ias.u-psud.fr, Telephone: (33)169 858575

${ }^{*}$ The NULLTIMATE Consortium is composed of the institutions where authors are affiliated plus IMEP and Institut Fresnel 


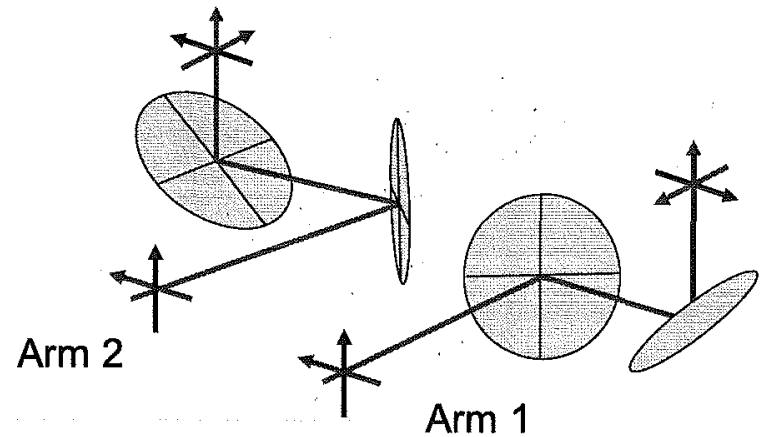

a)

Arm 1 : Triple Flat

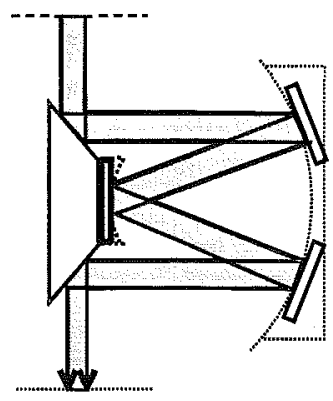

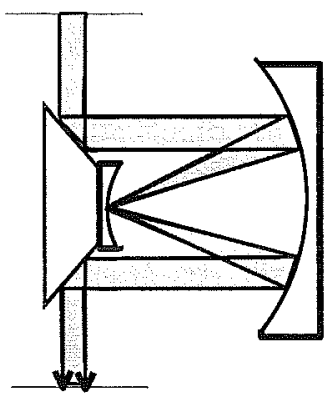

Arm 2 : Cat's Eye b) Prisms

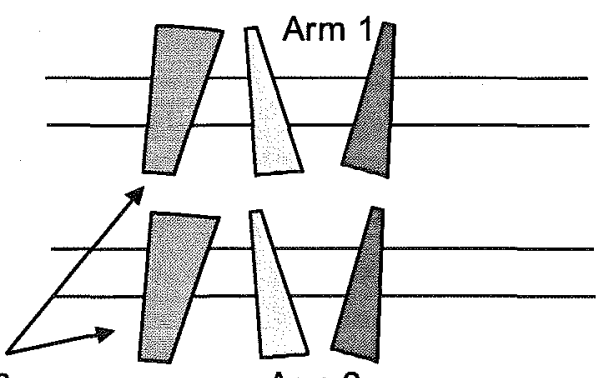

Arm 2

c)

Figure 1. Schema of the 3 Simulated APS: a) Field Reversal APS, b) Dispersive Plate APS, c) Focus crossing APS

\section{MODELING METHODS}

The simulation of APS differ from usual optical simulation by the high resolution needed to predict the performance of the devices. The interest of the work is the simulation of the physical environment of the three devices, in terms of vibration and temperature.

\subsection{Optical modeling}

The performance of the APS is expressed as the rejection rate $\rho=\frac{I_{\max }}{I_{m} i n}$ or nulling ratio $n=\frac{1}{\rho}$. This parameter gives the level of contrast achievable by the nulling interferometer. The typical Darwin scientific specification is $\rho=10^{4}-10^{5}$. However ESA requires to try to reach $10^{6}$. This means that for any optical calculation the resolution of the method should offer a dynamic better than $10^{6}$. This is impossible to find a single optical simulation method that would take into account all the necessary parameters and phenomena. Therefore the choice has been made to split the different phenomena into different models. To gather all the result the method used is the one described in Serabyn. ${ }^{2}$ The choice is to suppose that the contribution of each optical defect is independent. This might be a very restrictive assumption and we hope to be able to verify it experimentally

$$
\frac{1}{\rho^{2}}=N^{2}=\sum_{\text {defects }} n_{\text {defects }}^{2}
$$

The intended scheme and parameters of the modeling is given on figure 2 . In the following paragraphs I will describe the different models and parameters. Most of the models used to perform the simulation are provided by a commercial optical software, however new developments are still necessary. 


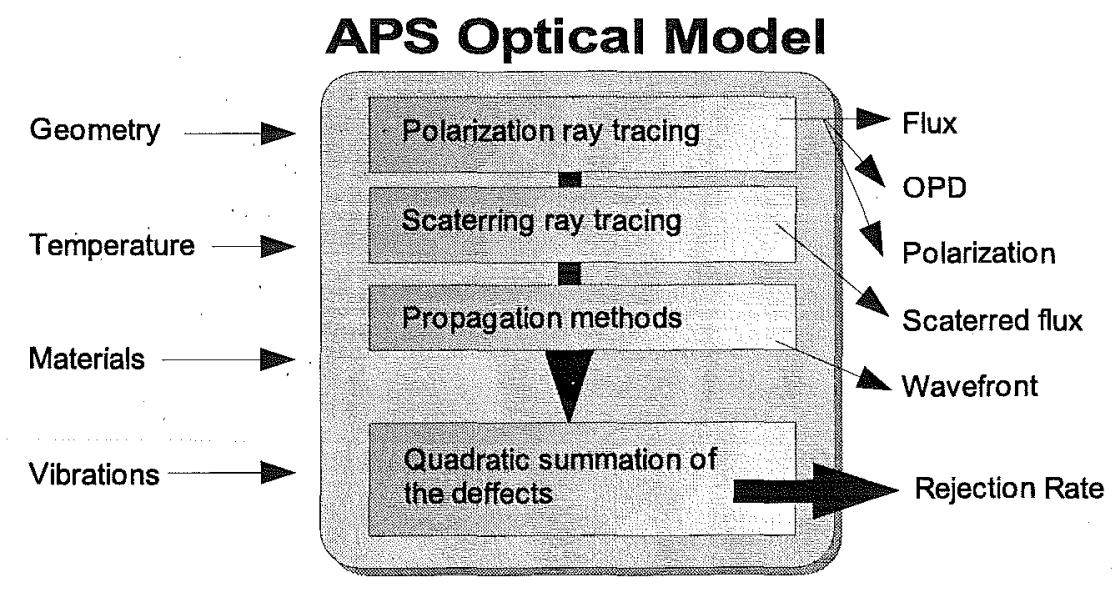

Figure 2. Illustrates the principle of the simulation performed

\subsubsection{Ray Tracing}

This model is based on geometrical optics. it can be extended to take into account the polarization of light. With one single ray on the optical axis of the optical devices, it is possible to derive the polarization state at the output of the interferometer. With several rays it is possible to compute the wavefront error. The model can also calculate flux, using massive ray tracing. This mode has however a poor accuracy : sending a million rays leads only to an accuracy of typically $3 \%$. this is why the scattering ray tracing is needed.

\subsubsection{Scattering ray tracing}

The optical surfaces of the different APS will be well polished. Their surface roughness will be of the order of the nm. Thus the total flux scattered by an optical surface is given by the TIS :

$$
T I S=\frac{4 \pi \Delta^{2} / 4}{\lambda^{2}} I_{i} n c i d e n t
$$

Where $\Delta$ is the rms roughness in nanometer. This flux is emitted in all directions. At $6 \mu \mathrm{m}$ this flux is $1.4 \times 10^{-6}$ the incident flux. However the detector will see a very tiny part of this flux, because of the small beam etendue seen by the detector. The contribution of the scattered flux on the rejection rate is direct :

$$
\frac{1}{\rho}=n_{\text {scatter }}=\frac{I_{\text {scatter }}}{I_{\max }}
$$

Thus the scattered intensity seen by the detector should be below $10^{-6}$ of the maximum transmitted flux.

In the software scattering analysis is also based on geometrical optics, however here when a ray encounter a surface its new direction is calculated statistically according to the chosen scattering law. The scattering flux could be evaluated at the same time than the general flux, however this is not possible due to the accuracy, and resolution needed. This part of the simulator is not ready, but the first order calculation are reassuring on the impact of scattering on the rejection rate. This part of the simulator will be however precious to analyze the scattering by non optical surface on the bench. 


\subsubsection{Propagation methods}

One of the most important parameter that has to be simulated is the wavefront. Two phenomena should be modeled :

- Diffraction

- Shape position and surface defects of different origins

In theory, it should be possible to treat both phenomena at the same time with a beam propagation algorithm. But usually propagation methods are quite heavy. Thus to ease tolerancing and dynamical simulation, it seems preferable to dissociate the two aspects. This means that we do the hypothesis that the diffraction effects are weakly dependent from the shape and position defects of the optics. These defects are tackled by ray tracing. The algorithm research is probably not complete, but we focused on a first working solution. The diffraction part of the software is not used in this paper.

\subsection{Tolerancing/Static Modeling}

The first step of the simulation is to model the built-in defects and the defects that have a slow variation with time, such as temperature.

\subsubsection{Built-in defects}

To estimate the effects of the built-in defects such as misalignment or shape imperfection, it is necessary to compute the modification of the wavefront error as a function of numerous parameters. Thus to reduce calculation time, Monte-Carlo methods are used.

\subsubsection{Temperature effect modeling}

The modeling of temperature effects on the optical parameters was done by a first order analytical approach. A finite element analysis is possible but probably extremely dependent of the future environment of the test bench, that is not completely defined at the time being. The thermal environment we intend to simulate is the one of the future test bench. This means a temperature of $100 \pm 1^{\circ} \mathrm{K}$. Temperature has two main consequences : dilatation/retraction of the different materials, and change in the refractive index. This last point is much sensitive for the APSDP. One of the important point to assess is the effect of thermal gradients (e.g index gradient) on the performance of the APS.

The main problem is the poor accuracy available on the property (if known) of the selected materials at the temperature of the test bench.

The method used to perform the simulations is the same than with built-in defects.

\subsection{Dynamic Modeling : Vibrations}

The modeling of vibration effects on optical parameters was performed two ways :

- A first order analytical approach

- A finite element analysis of each device

The input of this modeling were defined with the measure done on the IAS $2 \mu \mathrm{m}$ nulling bench (in these proceedings Brachet et $\mathrm{al}^{3}$ ). They are, we hope, a good approximation of the future experimental conditions on the NULLTIMATE test bench : the amplitude of the translation movements are about $20 \mathrm{~nm}$ in every direction. The rotations have not been measured thus they are considered to be the consequences of the translation vibration according to the geometry of the mounts on the bench. This gives an amplitude of rotation around the tenth of arcsecond. The spectrum of the excitation is approximatively white below $200 \mathrm{~Hz}$.

The first conclusion of this modeling is that vibration has no optically noticeable effects on the internal geometry of the mirror APS (e.g Focus Crossing and Field reversal APS). Thus the effects of vibration on these 


\begin{tabular}{lr}
\hline APSFC & \\
Specification & Tolerance \\
\hline Global Position & $0.1 \mathrm{~mm}$ \\
Focal Distance & $1 \mu \mathrm{m}$ \\
Surface Quality & $\frac{\lambda}{30}$ \\
Angular Direction & 2 arcsec \\
\hline
\end{tabular}

\begin{tabular}{lr}
\hline APSFR & \\
Specification & Tolerance \\
\hline Global Position & $0.1 \mathrm{~mm}$ \\
Surface Quality & $\frac{\lambda}{30}$ \\
Angular Direction & 40 arcsec \\
\hline
\end{tabular}

Table 1. Tolerances of the Field Reversal APS and Focus Crossing APS used for the simulations. These tolerance are not definitive, they are here only given as numerical values

APS are global movements inside the interferometer. For the dispersive plate APS, as the different plates are mechanically independent, the fact that distances and angles vary with vibrations cannot be avoided. For all the APS the vibration level is sufficiently low to avoid deformations of the optical surfaces.

One should notice that the deformation / movement of the optical system due to vibration are very low compared to misadjustments that are built-in. This remark intend to justify the choices made for the dynamic modeling. To get a precise image of the performances of the studied device, it is necessary to explore a mathematical space with lots of dimensions. The different positions and rotations, the different shapes errors, and the different movements. A regular sampling of this space would mean a too huge number of calculations. Thus dynamical parameters are modeled independently from the built-in defects. This limits the parameter space to 6 ( 3 translations, 3 rotations) for the mirror APS, and to 9 (3 translations, 6 rotations) for the dispersive plate APS. This represents still a large number of parameters. However their variation domain is very small. So inspired by multi-factorial analysis techniques ${ }^{4}$ we try do model each output of the model as first order polynomial evaluation of the parameter :

Let $\left\{x_{1} \ldots x_{n}\right\}$ be the parameters and $y$ one of the model output then

$$
y=\sum a_{i} x_{1}+\sum_{i \neq j} b_{k} x_{i} x_{j}+\sum_{i \neq j \neq k} c_{l} x_{i} x_{j} x_{k}+\ldots+\prod x_{i}
$$

The different coefficients of this model can easily be computed by a calculation of $y$ on each corner of the parameters space hypercube.

The use of such a model enables then numerous quick calculations, and thus to take into account the spectral composition of the mechanical excitation and the effect of the closed loop. Further more, first calculations seem to give a good confidence on its reliability : rms errors are around $10^{-7} \mathrm{rad}$ which is a negligible null contribution.

\section{RESULTS}

The result shown here are still preliminary : As a matter of fact the development of the simulation software is much slower than what was foreseen.

\subsection{Mirrors APS}

The two mirrors APS have in common to be made of bulk Zerodur coated by gold. The different pieces are bonded thank to molecular adhesion.

\subsubsection{Static Modeling}

Wavefront APSFC and APSFR were simulated with the tolerances given in table 1.

The result presented here are based on wavefront ray tracing. The wavefront is then projected on the Zernicke polynomial base (up to the $30^{\text {th }}$ ). The piston mode is discarded, because it can be compensated by delay lines. Tilt is not considered as causing directly a phase defect. Tilt can be compensated by the rotation of one mirror in the interferometer. This has however a performance cost that can be expressed as in equation 4 . This contribution rejection rate is not decreased by the wavefront filtering.

$$
n_{t i l t}=\frac{\left|\frac{d r_{s}}{d \theta}\right|^{2}+\left|\frac{d r_{p}}{d \theta}\right|^{2}}{\left|r_{s}\right|^{2}+\left|r_{p}\right|^{2}} d \theta^{2}
$$



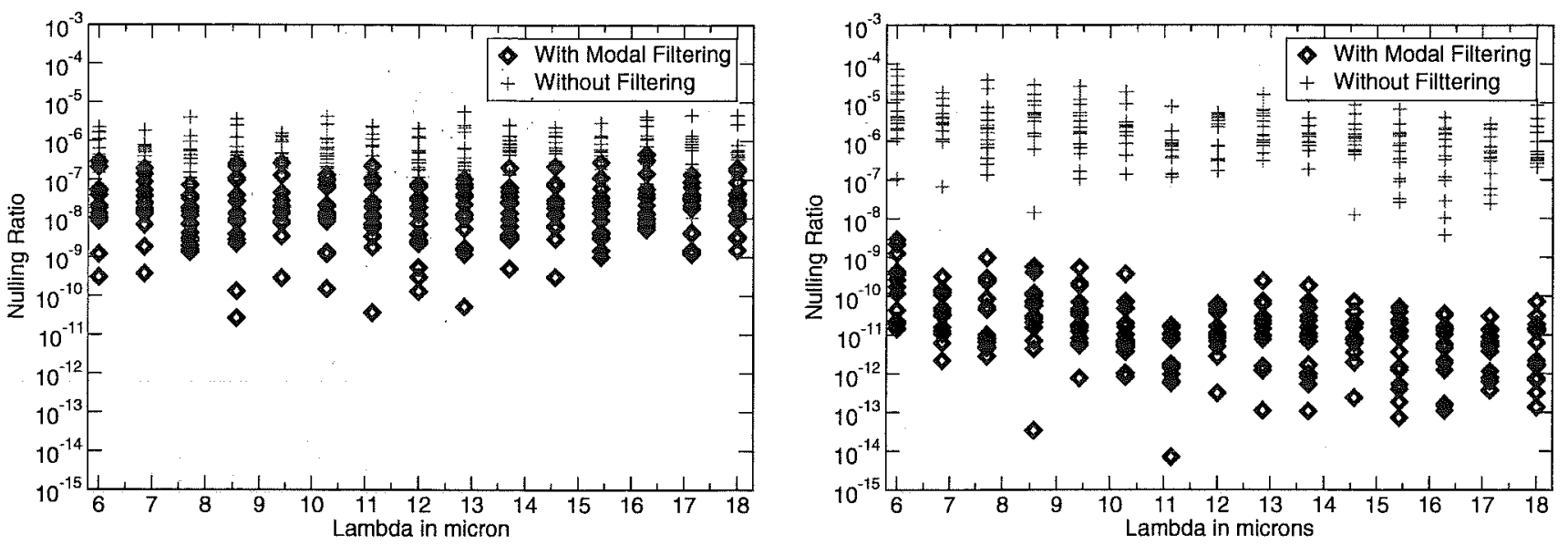

Figure 3. Monte-Carlo simulation of the two mirror APS by wavefront ray tracing, with built-in tolerances as described in table 1. Both simulation do not take piston into account, and tilt is added through the method of equation 4 . The difference in performance is linked to the impact of the tilt. Performance of the Focus crossing APS should decrease when all parameters will be taken into account

Field reversal APS

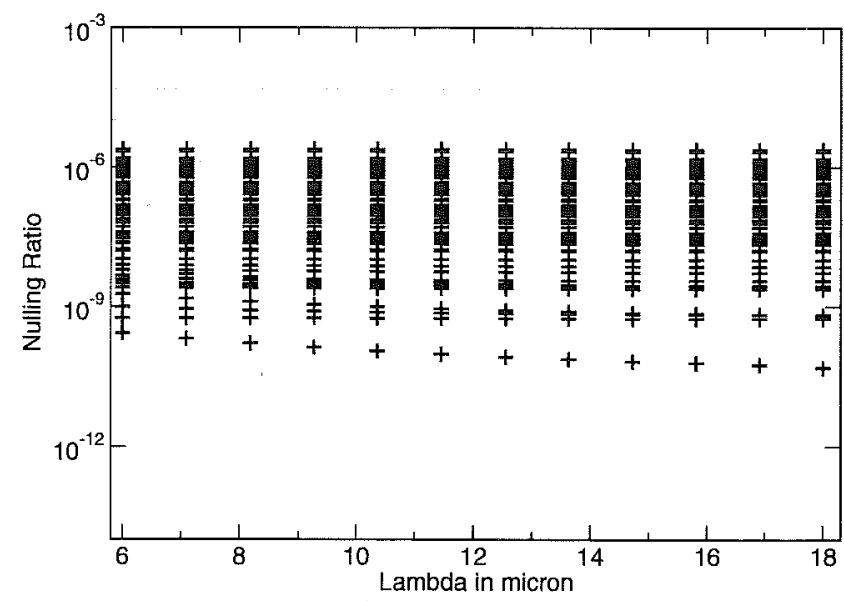

Focus Crossing APS

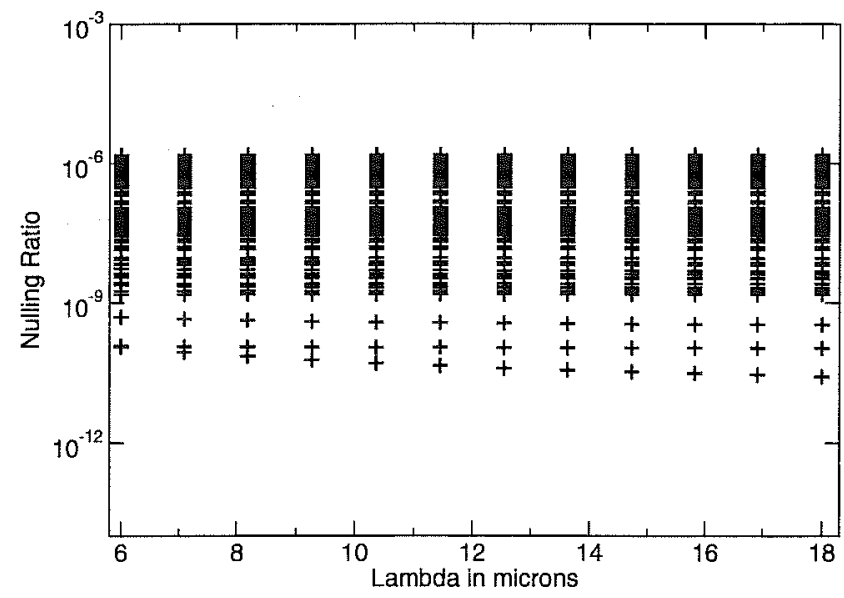

Figure 4. Monte-Carlo polarizsation simulation of the two mirror APS by the Jones Formalism, with built in tolerances as described in table 1 .

The wavefront error simulation results are shown on the figure 3 . As awaited the results are quite achromatic. For these simulations, the mirrors could have only one defect, which is curvature. For the case of the APSFC, the incidence dispersion problem is not taken into account, and the built-in defects are not completely modeled for technical reasons.

Polarization The polarization module of the simulation software is not functional. However, we present here results on polarization modeling obtained by the Jones Formalism (see figure 4). For these computations a single ray is sent into the system. These results were also obtained by Monte-Carlo methods. Polarization mismatch is one of the important contributors to the nulling ratio in this type of APS. One of the clear conclusions is that the more mirror we use the less built-in defects are acceptable on the orientation of the optical surfaces. This is illustrated the tolerance difference between the two mirror APS. An important conclusion is that polarization effects are quasi achromatic. 


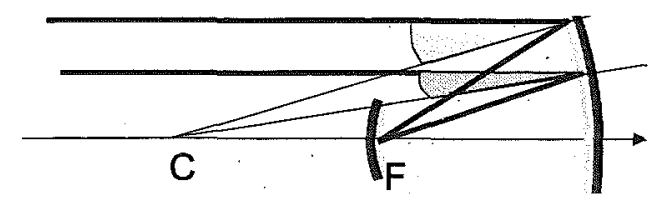

Cat'eye Chanel

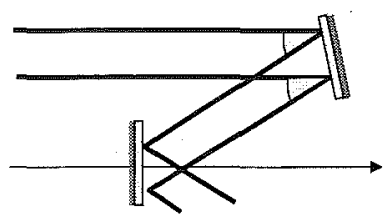

Triple Flat Chanel

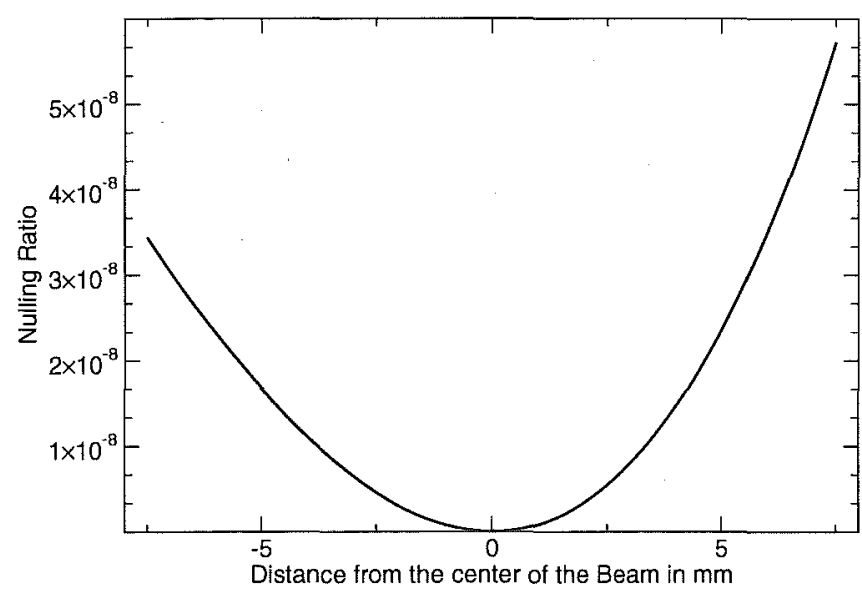

Figure 5. Illustration of the incidence dispertion phenomenon and its impact on nulling ratio of the Focus Crossing APS

Field reversal APS

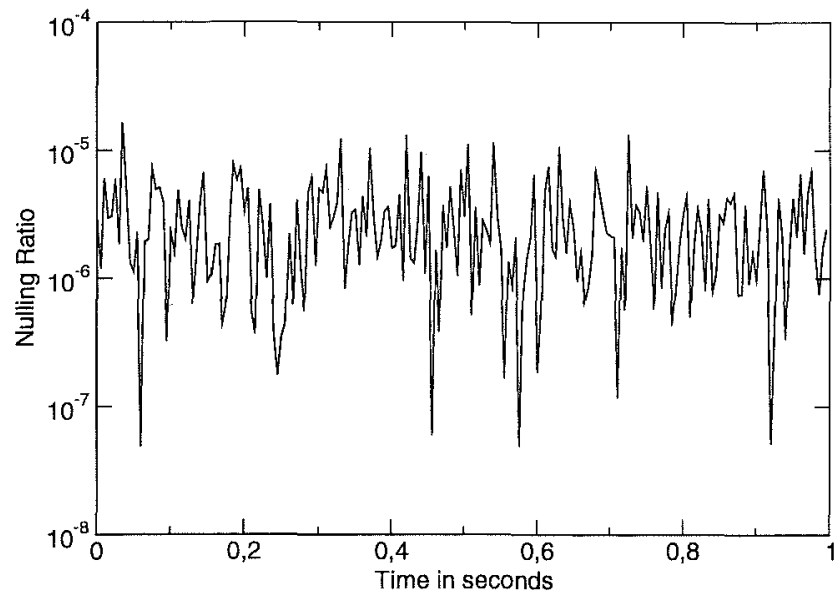

Focus Crossing APS

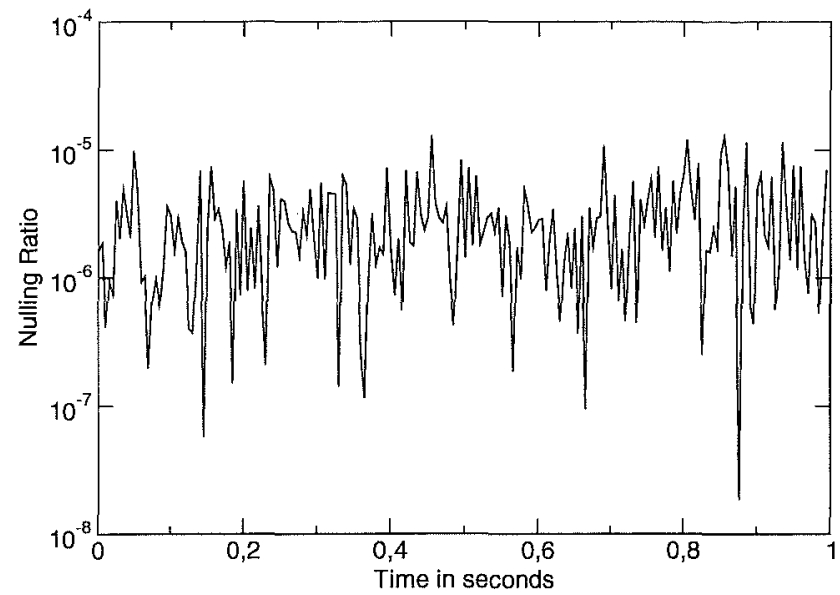

Figure 6. Temporal evolution of the nulling ratio due to vibrations, Both APS simulations take into account the built-in defects given in table 1 . However the curves represent only the vibration contribution to the nulling

The Focus Crossing APS has a special feature : There is a fundamental disymmetry in the optical setup. The beam impinging on the triple-flat train have a unique incidence, whatever the point of impact whilst the one impinging on the cat's eye experiences a spreading of the angle of incidence. This will have an impact on the rejection rate since the phase at reflexion is a function of the incidence. However numerical simulation show that this term is negligible (figure 5) the mean contribution is around for this design around $5 \times 10^{-8}$. The bigger the focal length of the primary mirror, the smaller the contribution to the nulling.

\subsubsection{Dynamic modeling}

The firsts dynamic computations shows the following results. The most important wavefront defects that are varying with vibration are piston and tilt. Piston can be corrected by the delay lines, tilt cannot. With the values taken for the computation which are $20 \mathrm{~nm}$ for translations and 0.1 ar sec for the rotation, the tilt contribution is of the same order than the corrected piston. The values taken for the rotation might be pessimistic, however we have until now no mean to evaluate it correctly. 


\begin{tabular}{lccc}
\hline Nulling contributions & APSFC & APSFR & APSDP \\
\hline Built-in WFE & $5 \times 10^{-9}$ & $4.6 \times 10^{-7}$ & TBD \\
Dynamical WFE & $3 \times 10^{-6}$ & $2.9 \times 10^{-6}$ & TBD \\
Built-in Polarisation mismatch & $2.3 \times 10^{-6}+$ incid disp $5 \times 10^{-8}$ & $2.4 \times 10^{-6}$ & $9 \times 10^{-8}$ \\
Diffusion & $\ll 10^{-6}$ & $\ll 10^{-6}$ & $\ll 10^{-6}$ \\
\hline Global Nulling & $3.8 \times 10^{-6}$ & $3.8 \times 10^{-6}$ & TBD \\
\hline
\end{tabular}

Table 2. Global predicted performances with the simulation tool in it's current state

\subsubsection{Thermal analysis}

The two mirror APS, are probably quite insensitive to the temperature variation :

- As they are made of a single material their shape changes in an homothetic way. The thermal expansion coefficient is quite low. From $300^{\circ} \mathrm{K}$ to $100^{\circ} \mathrm{K}$ the material contract itself, thus the optical quality of the device if temperature is homogeneous is conserved and even slightly bettered (a factor $1+10^{-5}$ ). At the working temperature, the thermal expansion coefficient is very low : $\alpha=1.8 \times 10^{-7 \circ} \mathrm{K}^{-1}$. If we consider a lateral thermal gradient of $1^{\circ} \mathrm{K}$ on a parallelpipedic Zerodur component of thickness $\mathrm{h}$ and height $\mathrm{L}$, the resulting deformation gives a tilt angle of :

$$
\frac{\Delta \theta=\Delta T \alpha L}{h}
$$

This gives for $L=20 \mathrm{~mm}$ and $h=20 \mathrm{~mm}$, a tilt angle $\Delta \theta=4 \times 10^{-2}$ arcsec. which is negligible compared to the built-in tolerances.

- The only uncertainty is the effect of the refractive index of the gold with temperature. There are however no available data on that last point. A quick analysis based on the Fresnel coefficient of a mirror at $45^{\circ}$ shows that to have a null of $10^{6}$, The imaginary index could vary of $1 \%$ and the real part of the index could vary of $5 \%$

\subsection{Dispersive APS}

The dispersive APS has not been simulated yet. Only the polarization contribution has been computed, the mean value is presented in table 2 .

\section{SUMMARY}

In this paper we have shown the methods that are implemented in the NULLTIMATE Test bench simulation software. This simulation tool is a challenging problem because of the number of the parameter to take into account and the high resolution needed. The preliminary of the APS performance obtained through our simulations can be seen in the table 2 . They show the estimated difficulty to achieve very high nulling.

\section{ACKNOWLEDGMENTS}

This work has been done under The ESA contract $\mathrm{N}^{\circ} 17005 / 02 / \mathrm{NL} / \mathrm{JA}$. It would not have been possible without the help of the whole NULLTIMATE CONSORTIUM e.g : Alcatel Space, OCA, Kayser Threde ...., the PhD grant of the author (B.C.) is funded by CNRS and ALCATEL SPACE Industries.

\section{REFERENCES}

1. A. Labèque, B. Chazelas, F. Brachet, C. Commeaux, P. Blache, A. Léger, T. Lépine, M. Ollivier, and V. c., "Low temperature nulling interferometer test bench for Achromatic Phase Shifters," in SPIE Vol 5491, Astronomical Telescopes and Instrumentation, Adrian Russell, Roberto Gilmozzi; Eds., 2004. 
2. E. Serabyn, "Nulling interferometry: symmetry requirements and experimental results," in Proc. SPIE Vol. 4006, p. 328-339, Interferometry in Optical Astronomy, Pierre J. Lena; Andreas Quirrenbach; Eds., pp. 328339, July 2000.

3. F. Brachet, A. Labèque, A. Léger, M. Ollivier, C. Lizembert, V. Hervier, B. Chazelas, T. Lépine, and C. Valette, "Nulling interferometry for the Darwin mission polychromatic laboratory test bench," in SPIE Vol 5491 ,Astronomical Telescopes and Instrumentation, Adrian Russell, Roberto Gilmozzi; Eds., 2004.

4. J. Goupy, Introduction aux plan d'expérience, Technique et Ingénierie : Conception, Dunod, second ed., 2001. 